\title{
Reflets
}

Revue ontaroise d'intervention sociale et communautaire

\section{Stratégies de survie et d'identité : les dynamiques culturelles dans un projet d'intervention en prévention communautaire}

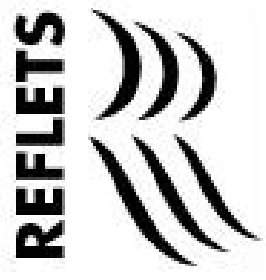

\section{Lamine Diallo et Marge Reitsma-Street}

Volume 1, numéro 1, printemps 1995

Des pratiques à notre image : défis et ressources

URI : https://id.erudit.org/iderudit/026054ar

DOI : https://doi.org/10.7202/026054ar

Aller au sommaire du numéro

Éditeur(s)

Reflets : Revue ontaroise d'intervention sociale et communautaire

ISSN

1203-4576 (imprimé)

1712-8498 (numérique)

Découvrir la revue

Citer cet article

Diallo, L. \& Reitsma-Street, M. (1995). Stratégies de survie et d'identité : les dynamiques culturelles dans un projet d'intervention en prévention communautaire. Reflets, 1(1), 43-69. https://doi.org/10.7202/026054ar
Résumé de l'article

Le projet « Partir d'un bon pas vers un avenir meilleur », conçu et mis sur pied à Sudbury, offre l'exemple d'un travail de collaboration entre la communauté minoritaire franco-ontarienne et d'autres communautés culturelles, axé sur le respect des différences, l'autonomie et la valorisation de la culture. Ce texte présente trois étapes clés du processus de développement de ce projet, et analyse les tensions qui ont été soulevées et qui ont contribué à la définition de la structure actuelle du projet.
Tous droits réservés (C) Reflets : Revue ontaroise d'intervention sociale et communautaire, 1995
Ce document est protégé par la loi sur le droit d'auteur. L'utilisation des services d'Érudit (y compris la reproduction) est assujettie à sa politique d'utilisation que vous pouvez consulter en ligne. 


\section{Stratégies de survie et d'identité:}

\section{Les dynamiques culturelles dans un projet d'intervention en prévention communautaire}

Le projet "Partir d'un bon pas vers un avenir meilleur», conçu et mis sur pied à Sudbury, offre l'exemple d'un travail de collaboration entre la communauté minoritaire franco-ontarienne et d'autres communautés culturelles, axé sur le respect des différences, l'autonomie et la valorisation de la culture. Ce texte présente trois étapes clés du processus de développement de ce projet, et analyse les tensions qui ont été soulevées et qui ont contribué à la définition de la structure actuelle du projet.

Lamine Diallo, M.A.

Chercheur pour le projet

Marge Reitsma-Street, Ph.D.

Directrice de l'École de service social, Université Laurentienne et Consultante pour le projet

\section{Introduction}

Partir d'un bon pas pour un avenir meilleur est un projet provincial longitudinal en prévention communautaire. Il cherche à mettre en évidence certaines initiatives plus aptes à contribuer à la réduction des problèmes de comportement et de pauvres résultats scolaires liés aux facteurs de fragilisation des communautés à faible revenu (ministère des Services sociaux et communautaires, 1989). 
Reflets

Le projet, d'une durée de cinq ans, favorise pour cette expérience différents éléments dans sept communautés urbaines et quatre réserves autochtones en Ontario.

Ce texte examine le processus par lequel la culture francophone a d'abord été définie comme un contexte d'intervention dans le projet Partir d'un bon pas pour un avenir meilleur de Sudbury, pour ensuite se transformer en objectif majeur autour duquel s'articule le modèle d'intervention communautaire dans les quartiers défavorisés.

En 1988, le ministère des Services sociaux et communautaires publie le document de consultation "Investir dans l'enfance: nouvelles orientations relatives au traitement de l'enfant et à l'intervention en faveur de la famille», qui établissait l'importance de la prévention chez les populations à risque élevé (Peters \& Russel, 1994). Plusieurs autres recherches avaient déjà démontré que la pauvreté ne diminuait pas pour les enfants et les familles au Canada ou en Ontario. Selon le recensement de Statistique Canada, le pourcentage d'enfants vivant dans des familles à faible revenu en 1986 était de 19,8 \% pour la ville de Sudbury. Cinq ans plus tard, ce pourcentage avait connu une légère hausse, pour atteindre 21,4 \% (Carrière et al., 1992; Hein, 1994). Ce pourcentage est sensiblement plus élevé que celui du reste de l'Ontario, qui se situe à 14,7 \%, et il est presque le double de celui de l'Europe de l'Ouest (Carrière et al., 1992; Hein, 1994). Pour certains groupes, notamment les enfants de moins de six ans nés de mères adolescentes, les enfants de familles mono-parentales et les enfants qui vivent dans certaines communautés géographiques ou culturelles, le pourcentage est même plus élevé que $20 \%$ (Reitsma-Street et al, 1993; Johnson, 1983).

Dans les foyers à faible revenu, la situation est encore plus sérieuse et d'une plus longue durée, surtout lorsqu'elle est accompagnée d'autres problèmes tels que le surpeuplement des écoles et le stress chez les parents (Rutter et Madge, 1976). Les enfants et les adolescents provenant de foyers à faible revenu sont aussi plus vulnérables aux autres types de problèmes tels que les mauvais traitements, les problèmes de santé mentale, la faible performance et l'abandon scolaire. L'étude longitudinale sur la 
«...le Canada... compte parmi les pays ayant les taux les plus élevés de pauvreté infantile.» santé mentale des enfants de l'Ontario avait en effet révélé qu'un enfant sur six présentait un trouble affectif ou comportemental identifiable, et que les familles bénéficiant de l'aide sociale ou habitant un logement subventionné risquaient davantage de connaitre ces problèmes (Offord \& al., 1987; Lipman \& al., 1994; Kitchen et al, 1991; Volpe, 1989).

Plusieurs études ont démontré que l'approche individuelle ou familiale pour réduire ou prévenir la pauvreté et ses conséquences est moins efficace que l'approche communautaire, l'adoption de politiques sociales ou l'approche axée sur le marché du travail. McFate (1991) et Smeeding (1988-89), comparant le taux de pauvreté et son impact dans différents pays, ont trouvé que les pays qui adoptent des politiques sociales telles que des allocations familiales substantielles, des systèmes de garderie universels, une éducation sexuelle intensive et des programmes scolaires prématernels ont les taux de pauvreté des plus faibles. Ils ont aussi constaté que le Canada est en voie de rejoindre les ÉtatsUnis et compte parmi les pays ayant les taux les plus élevés de pauvreté infantile.

Le modèle de «Partir d'un bon pas pour un avenir meilleur» est axé sur une approche communautaire et n'identifie pas les familles fragilisées à cause d'un faible revenu ou de la structure familiale. Ce modèle met l'accent sur l'ensemble de la communauté, particulièrement les enfants de zéro à huit ans et leurs familles, en se penchant sur le voisinage, le quartier ou les écoles ayant des taux élevés de pauvreté, de chômage ou un faible niveau d'éducation parentale (Rutter 1983; Comer 1980).

L'approche d'intervention communautaire utilisée permet d'identifier les besoins de la communauté, ainsi que les forces et les obstacles au changement. Par la mise sur pied d'une coalition de résidents et résidentes, et avec la collaboration de divers services sociaux, des programmes communautaires universels sont développés et mis en oeuvre par une structure locale. Ce modèle innovateur donne aux communautés choisies la liberté de décider du type de programmes qu'elles veulent développer.

Les liens entre l'intervention et les taux élevés de foyers à faible revenu parmi certains groupes culturels au Canada, 
Reflets

"Les liens entre

l'intervention et les

taux élevés de foyers à

faible revenu... n'ont

pas encore été docu-

mentés.» particulièrement chez les autochtones, les nouveaux immigrants et certains francophones résidant dans le nord (Dennie, 1978), n'ont pas encore été documentés. Il est donc impossible d'établir s'il existe ou non un lien entre l'identité culturelle ou son absence, et les méthodes de prévention communautaire ou d'intervention. Toutefois, dans son approche, le projet Partir d'un bon pas a mis l'accent sur un modèle intégré et a sélectionné des sites variés au niveau socio-culturel et géographique, en choisissant des réserves autochtones, des communautés francophones et plusieurs sites multiculturels urbains.

Au début du projet, la force, les contradictions et les impacts de la culture ou de la race n'étaient pas jugés essentiels dans l'approche de ce modèle intégré. Ces facteurs se sont avérés presque invisibles dans le premier rapport sur l'historique et les premières années de vie des sept sites urbains (Rapport non encore officiel). Aussi, peu nombreuses sont les questions se rapportant à la culture et à son rôle dans les principaux instruments de mesure utilisés dans la cueillette des données, soit les entrevues avec les parents et les tests avec les enfants.

Cependant, après trois ans d'activité, nous sommes en mesure de faire quelques commentaires sur les manifestations de l'émergence de la culture et de la race comme éléments importants de l'intervention communautaire dans les différents sites, plus particulièrement celui de Sudbury. Nous présenterons trois stades qui marquent le développement de la culture dans le projet de Sudbury. Sans tirer de conclusions définitives, nous proposerons certaines leçons à retenir et nous soulèverons des questions et des préoccupations liées à cette expérience.

\section{Méthodologie}

Cette recherche se déroule depuis le début du projet Partir d'un bon pas pour un avenir meilleur en décembre 1989 et s'échelonne jusqu'en avril 1994. Les sources d'information utilisées comprennent: les écrits sur le sujet, les notes des réunions hebdomadaires de l'Association Partir d'un bon pas de Sudbury (organe décisionnel du projet), cinq entrevues en profondeur avec la coordinatrice 
du projet, une autre entrevue avec deux des membres fondateurs du groupe francophone, une entrevue de groupe avec des membres de la communauté francophone participant au projet, les comptes rendus d'ateliers et divers documents connexes ainsi que les observations, les notes et les réflexions des chercheurs engagés dans le projet.

\section{Description du projet Partir d'un bon pas de Sudbury}

Lors de sa création, le projet vise trois principaux objectifs:

- prévenir chez les jeunes enfants les graves problèmes sociaux, affectifs, comportementaux, physiques et intellectuels;

- promouvoir chez les enfants le développement social, affectif, comportemental, physique et intellectuel;

- rendre les familles et les milieux socio-économiquement défavorisés plus aptes à pourvoir aux besoins de leurs enfants.

Le projet est subventionné par trois ministères du gouvernement de l'Ontario (Services sociaux et communautaires, Santé et Éducation) ainsi que le ministère fédéral des Affaires indiennes et du Nord et le Secrétariat d'État. Il s'agit d'un des premiers projets pilotes au Canada en matière de recherche sur les politiques de prévention à long terme.

Sudbury fait partie des onze localités différentes de l'Ontario choisies pour les projets. Les quartiers sélectionnés sont situés dans des zones socio-économiquement défavorisées, et la période de mise en oeuvre est de cinq ans avec un suivi des groupes cibles jusqu'à l'âge de 25 ans. Les groupes ciblés sont les enfants de 0 à 4 ans et leur famille pour certains sites, et ceux de 4 à 8 ans pour d'autres sites; Sudbury fait partie de cette seconde catégorie.

Le site qui a été choisi se situe dans les quartiers de Donovan et du Moulin à Fleur, bastion de la communauté francophone de la ville de Sudbury (30 \% de la population). On retrouve aussi dans 
Reflets

«Les caractéristiques

de la population

francophone concernée nous permettent de proposer des liens entre pauvreté et culture.» ces quartiers une forte communauté autochtone (10\%) et multiculturelle. Le recensement de 1986 montre qu'environ un enfant sur trois de ces deux quartiers vit dans une famille désavantagée; par exemple, 30,3\% des familles se retrouvent audessous du seuil minimum de pauvreté défini par Statistique Canada, comparé à 14,8\% pour la région et $11 \%$ pour la province (Statistique Canada, 1986). Le revenu moyen dans cette communauté, en 1986, était légèrement supérieur à 15000 \$33000 \$ au niveau provincial). Environ un enfant sur quatre vit dans une famille recevant de l'aide sociale (7,9\% au niveau provincial), et $43 \%$ des enfants de moins de 9 ans vivent dans des logements subventionnés.

Les caractéristiques de la population francophone concernée nous permettent de proposer des liens entre pauvreté et culture. Au sein de la communauté francophone du Nord de l'Ontario, les personnes qui vivent dans les centres urbains tel Sudbury sont exposés aux effets de l'assimilation et de l'isolement. À Sudbury, le déplacement vers la périphérie des francophones mieux nantis rend plus vulnérables les francophones des quartiers du Moulin à Fleur et Donovan, comme l'explique un des membres du projet:

"Ce sont les gens qui habitent d'autres quartiers..., vus comme des personnes au revenu élevé, qui se moquent des faibles revenus des autres, il $y$ a des gens pauvres qui habitent ce quartier mais il $y$ a des gens qui réussissent. L'étiquette vient de la base, de la fondation même du quartier, la séparation des francophones avec la création d'une nouvelle paroisse au centre ville... Pour ne pas être identifiés comme pauvres et pour des raisons de logement, les jeunes francophones quittent le quartier..."

L'intervention communautaire dans le projet de Sudbury débute à l'automne 1990 suite à l'enquête menée auprès de plus de 300 parents, enfants et membres d'organismes sociaux pour décider des besoins et des désirs des résidents et résidentes. Cette enquête a permis de redéfinir les trois objectifs gouvernementaux mentionnés plus haut et de les transformer en quatre objectifs locaux 
concrets et réalisables (Sudbury Better Beginnings, Better Futures Proposal, 1989). Les besoins communautaires s'articulent autour des quatre points suivants:

1. Une bonne éducation pour les enfants et l'accroissement des possibilités d'éducation postsecondaire.

2. Des lieux d'enseignement sûrs et accessibles.

3. Un centre de rencontre où les adultes et les enfants peuvent jouer, apprendre et partager.

4. La création d'activités pour aider les enfants francophones et amérindiens à développer leurs identités culturelles.

Le modèle de Sudbury cible la rénovation et la réouverture d'anciens centres communautaires pour créer des lieux de rencontre sécuritaires pour les parents et les enfants et soutient la réorganisation des associations communautaires telles que les associations de parents dans les écoles et les associations des locataires dans les logements publics. Le modèle appuie aussi l'éducation communautaire par des ateliers offerts aux résidents, la défense des intérêts des résidents par l'encadrement et le support matériel, et la création d'une atmosphère plus favorable aux études dans les écoles par la distribution de nourriture, l'organisation de jeux coopératifs et l'éducation culturelle.

\section{Les trois stades de développement du projet}

La présentation des trois stades de développement démontrera l'impact de l'approche culturelle et illustrera la structure et le fonctionnement développés par le projet pour respecter la nature multiculturelle du site. Nous analyserons aussi, à la lumière du groupe francophone, les tensions, les incidents critiques, les difficultés et les conflits survenus lors du développement de la structure. Nous verrons également l'impact des facteurs de risque sur l'approche multiculturelle.

Ces données permettront une meilleure compréhension des stratégies du groupe francophone pour atteindre l'objectif de 
Reflets

protection et de sauvegarde de sa culture. Les éléments intégrés dans la programmation pour développer la fierté francophone chez les enfants du site seront aussi décrits.

\section{L'approcheinterculturelleetle développement du groupe francophone}

Au début du projet, le groupe constitutif était composé de représentants délégués par huit agences et organismes locaux qui se sont regroupés en Association pour rédiger la demande initiale qui a été soumise au gouvernement (The Sudbury Better Beginnings, Better Futures proposal, juillet 1990). Pendant cette période, la présence francophone était minime, mais certains organismes étaient représentés par des personnes francophones.

Le respect des différentes cultures et le fait de vouloir leur donner l'occasion de se développer dans le cadre du projet sont alors retenus comme éléments essentiels dès le printemps 1990. Le groupe constitutif commence à déployer des efforts pour encourager la participation communautaire, surtout du côté francophone. L'approche interculturelle vise à favoriser l'autonomie et la participation des groupes culturels dans la définition des objectifs et des programmes. La présence de trois cultures autonomes (anglaise, francophone et amérindienne) et d'une forte communauté multiculturelle (Africains, Latino-américains, Jamaïcains et Arabes) dans le site justifie le choix de cette approche.

Dès sa naissance, l'Association (organe décisionnel du projet) décide de fonctionner dans les deux langues officielles afin de faciliter la participation des francophones. Plusieurs efforts sont

"...l'Association travaille au développement d'une structure permettant aux groupes culturels de se réunir séparément pour discuter dans leur langue des questions et des besoins qui leur sont propres.» déployés par les membres, à l'époque, pour promouvoir la nature bilingue de l'association. Tous les documents locaux sont rédigés dans les deux langues officielles et les postes «cadres» du projet sont désignés bilingues. Cependant, comme c'est souvent le cas dans de telles situations, le maintien du bilinguisme dans les réunions s'avère difficile, la plupart des membres étant unilingues anglophones.

Confrontée à ce défi, l'Association travaille au développement d'une structure permettant aux groupes culturels de se réunir séparément pour discuter dans leur langue des questions et des 
besoins qui leur sont propres. Dans cet optique, au printemps 1991, plusieurs réunions ont pour objectif de préciser les rôles, le fonctionnement et les relations entre les différents «groupes de travail» (caucus) qui seraient créés. Cet effort amène la création de quatre caucus, francophone, autochtone, anglophone et multiculturel dès février 1991. Le caucus multiculturel regroupe toutes les cultures autres que celles mentionnées précédemment. À cause de sa composition hétérogène, ce caucus demeure très peu actif durant les premières années du projet. L'Association accepte aussi quatre mois plus tard de créer un caucus "professionnel» composé de représentantes et représentants des divers organismes de la région, en plus du caucus de recherche qui existait déjà depuis 1990.

Les caucus devaient permettre aux groupes minoritaires des Francophones, des Autochtones et de la communauté multiculturelle de s'organiser et de travailler de façon autonome au développement de programmes adaptés aux besoins de leurs communautés. Ils devaient permettre aux minorités culturelles de pouvoir bénéficier d'une structure leur permettant de s'exprimer et de se faire entendre. Par ailleurs, le caucus de recherche et celui des agences n'avaient aucune représentation culturelle, mais le premier était ouvert à tous les résidents et l'autre à tous les représentants d'agences.

Les caucus culturels sont composés de résidents et résidentes bénévoles ainsi que des employés du projet et se réunissent séparément et de façon régulière, afin de consulter les membres de leur communauté. Ils prennent aussi des décisions sur les types de programmes et d'activités à offrir à leur communauté. Ces décisions sont ensuite présentées à l'association qui se réunit à chaque semaine en présence des membres des différents caucus, des employés du projet et de tous les bénévoles intéressés au projet. Les rapporteurs choisis par les caucus présentent les décisions de leur groupe pour approbation par consensus de l'association.

Cette période de mise en place d'une approche interculturelle se concrétisera grâce à une longue concertation dans le cadre de réunions hebdomadaires permettant aux membres de l'Association 
Reflets

"...certaines

incompréhensions sont

en train d'apparaître à cause de nos différences

culturelles, et ces

incompréhensions

peuvent être sources de

frustration.» d'établir les mécanismes de facilitation d'une compréhension et d'une collaboration accrue entre les différentes cultures.

En juin 1991, une série d'entrevues entre les membres de l'Association permet de déterminer les forces et les limites de l'approche interculturelle dans le projet. Le compte rendu des responsables de la recherche suite à ces entrevues démontre les tensions et les avantages liés à cette approche. Cet exercice donne aux membres l'occasion de discuter ouvertement de l'impact de l'approche interculturelle.

Quoique les membres se disent prêts à tenter de mieux se connaitre au niveau culturel pour atteindre des objectifs communs, la majorité de ceux interviewés reconnaissent l'existence de différences culturelles s'exprimant de diverses façons: le langage, l'habillement, la profession, le niveau de revenu et d'éducation entre autres. Même s'ils manifestent de nombreux préjugés, par exemple percevoir certaines cultures comme étant calmes ou non-communicatives, les membres font preuve de respect et d'un désir d'en apprendre davantage sur les autres cultures.

Par contre, certaines barrières deviennent évidentes, comme l'exprime clairement un membre: "certaines incompréhensions sont en train d'apparaitre à cause de nos différences culturelles, et ces incompréhensions peuvent être sources de frustration.»

D'autres membres affirment que le développement rapide du projet risque de laisser en marge certains groupes qui pourraient être d'avis que le projet ne leur donne pas assez d'espace et de sécurité pour s'exprimer librement. Il faut donc ralentir la procédure décisionnelle et prendre le temps d'écouter attentivement les propos des autres pour faciliter une meilleure compréhension.

L'approche interculturelle est aussi considérée comme une force, donc une façon d'assurer une protection et une affirmation non seulement de la culture francophone, mais aussi des autres cultures. Parce que cette approche a été appuyée par les membres de l'Association, les incertitudes que laisse entrevoir l'avenir sont plus acceptables. Néanmoins, les membres se demandent souvent comment l'Association maintiendra l'équilibre entre les différents objectifs de certaines cultures (langue, autonomie...) et les objectifs communs du projet (lutter contre la pauvreté et l'oppression). 
"Dès sa création, le caucus francophone s'anime d'une grande effervescence et bénéficie d'une importante participation de la part des leaders communautaires.»

"...une autre crise de développement... se manifeste avec le licenciement du premier travailleur communautaire francophone.»
Cette approche permet toutefois de renforcer l'implication communautaire au niveau des caucus. Dès sa création, le caucus francophone s'anime d'une grande effervescence et bénéficie d'une importante participation de la part des leaders communautaires. Cette activité est rendu possible grâce à l'embauche, dès le printemps 1991, d'un travailleur communautaire pour chaque groupe culturel. Un des rôles importants de ces travailleurs est d'encourager les résidentes et résidents à participer, par l'entremise des caucus, à tous les niveaux du développement du projet : le choix des programmes et des activités et l'embauche des employés du projet.

Pour le groupe francophone, combattre l'assimilation et assurer le respect de l'autonomie impliquent la nécessité de posséder un centre séparé pour offrir des programmes durant la première année du projet, un seul centre abrite les programmes de tous les groupes. Des négociations sont donc entamées avec certaines institutions, le projet et le groupe francophone pour obtenir un centre distinct. Grâce à une entente conclue avec un conseil scolaire, les locaux d'une ancienne école sont loués à cette fin. Rappelons que plus les francophones et les autres groupes culturels précisent leurs besoins, plus le risque de division des différentes communautés culturelles augmente.

La location d'un centre pour les francophones crée aussi quelques tensions en raison du coût. Mais suite à de nombreuses discussions, l'Association accepte, par consensus, de défrayer les frais de location. La tension provient du fait que le seul local utilisé à date ne coûte presque rien au projet, en raison d'une collaboration avec un organisme municipal. Un premier foyer de tension se centre donc sur le volet financier.

Au cours de l'hiver 1992, une autre crise de développement liée à l'approche interculturelle se manifeste avec le licenciement du premier travailleur communautaire francophone. Pendant cette période, une attitude de méfiance, pour ne pas dire défensive, au niveau de l'Association incite certains membres du caucus francophone à douter de l'impartialité de l'organisme autochtone qui parraine le projet Partir d'un bon pas. Des membres du groupe francophone sont d'avis que ladite structure est inapte à la prise de décisions dans cette situation. Les soupçons face à cet organisme 
Reflets

«Des tours de table permettent à chacun de s'exprimer..." de la part de certains francophones augmentent la tension entre les membres des différentes communautés culturelles. Cette situation entraîne des dissensions à l'intérieur du groupe francophone, tout en rendant visible une certaine fragilité dans les relations culturelles entre les quatres communautés culturelles. Un second centre de tension, plus aigu que le premier, gravite autour des compétences du personnel à l'égard de certaines prises de décision.

Pour faire face à ce problème, l'Association recommande la création d'un "comité du personnel» composé d'un représentant nommé par chaque caucus. Ce comité doit recevoir toutes les plaintes concernant la discipline des travailleurs et travailleuses et recommander les mesures appropriées. Cependant, pour plusieurs raisons telles, entre autres, la confidentialité, ce comité ne sera jamais efficace.

Peu après cet incident, un troisième point de discorde surgit lorsque les francophones manifestent leur malaise face à la procédure du circling in, du fait que cette pratique soit d'origine autochtone. Au printemps 1992, un membre de l'Association rapporte: «Certains membres du groupe francophone disent qu'ils ne sont pas à l'aise avec le circling in, ce n'est pas dans leur culture.»

Pour répondre à ce genre de difficultés, les membres de l'Association organisent des ateliers intensifs sur l'interculturalisme et l'antiracisme. Ces ateliers sont obligatoires pour les employés et le projet encourage aussi les résidents et résidentes à y participer. Ils ont pour but de permettre une meilleure compréhension de la culture des autres groupes participant au projet.

Quant au mode de prise de décision par consensus choisi au départ, il donne aux membres la possibilité de discuter librement et de prendre le temps nécessaire pour se comprendre et pour mieux évaluer l'impact des décisions à prendre. Ce modèle, inspiré de la communauté autochtone, offre à tous les membres de l'Association l'occasion de donner leur point de vue sur toutes les décisions. Des tours de table permettent à chacun de s'exprimer; s'il y a des positions contradictoires, une autre série de discussions s'ouvre pour favoriser une meilleure compréhension. Cependant, en cas de discordance totale, d'autres réunions 
"...malgré les tensions

et les problèmes

d'alors, aucun conflit

ouvert n'éclate et les

membres continuent...

à trouver des solutions

communes aux

difficultés qui font

surface.»

s'organisent jusqu'à ce qu'un point d'entente soit atteint; sinon, un comité de conciliation est mis sur pied pour favoriser le rapprochement des idées divergentes. Les membres ne sont pas obligés d'accepter la décision litigieuse, mais on tente d'obtenir l'appui de tous avant que la décision soit entérinée. Ce modèle axé sur la concertation sert à rapprocher les points de vue des divers caucus.

L'une des particularités de l'approche interculturelle consiste aussi à développer des procédures permettant aux différentes communautés culturelles de participer et de jouer un rôle important dans l'embauche des employés du projet. Les francophones peuvent donc participer à l'embauche de leurs travailleurs, mais ils peuvent aussi siéger sur les comités d'embauche des autres groupes culturels. Tous les groupes participent à l'embauche et à l'évaluation de la coordinatrice et du chercheur du projet.

Cependant, malgré les tensions et les problèmes d'alors, aucun conflit ouvert n'éclate et les membres continuent, dans le cadre des réunions de l'Association, à trouver des solutions communes aux difficultés qui font surface. Les ateliers sur les relations interculturelles se poursuivent et facilitent des discussions approfondies sur les questions culturelles. Lors de sa réunion du 28 avril 1993, l'Association recommande la recherche d'un consultant pour les aider à répondre à certaines questions:

«... Qui sont les porteurs de la culture? Qu'est-ce qui rend la culture réelle? Comment cela nous permet de travailler ensemble? Nous planifions un atelier vers la fin juin avec les employés d'été. Nous voulons étudier la culture pour voir comment nous faisons face à plusieurs choses: le stress, les valeurs dans cette culture, la famille, quelle est la responsabilité du voisinage, s'il existe? Nous voudrions identifier comment la culture est véhiculée dans la famille, qu'est-ce qui rend notre culture réelle? Le langage? La spiritualité? Un jeu de rôles va être suggéré pour identifier le conflit dans votre culture. D'autres suggestions : étudier la valeur de la communication dans la culture, le ton de la voix, les relations...» 
Reflets

\section{L'identité distincte et le rétablissement de la fierté francophone}

L'identité distincte recherchée par le caucus francophone, question soulevée durant les premières années du projet, se concrétise en mars 1992, avec l'obtention d'un centre séparé et la mise sur pied de programmes s'adressant aux francophones. L'ouverture de ce centre permet au groupe d'offrir des activités ayant un cachet linguistique et culturel spécifique. L'un des objectifs principaux du groupe est d'offrir aux enfants des activités en français, dans un contexte et avec des travailleurs francophones. Il faut savoir qu'auparavant, la langue de travail au niveau du personnel, et avec la population, était l'anglais.

"Le groupe franco-

phone parvient non

Le groupe francophone parvient non seulement à se faire entendre, mais à marquer sa différence par la nature de ses activités. Dans les programmes francophones, tous les employés sont francophones, ils et elles sont embauchés par des comités sur lesquels siègent des membres de la communauté francophone. Tous les programmes du projet sont gratuits et accessibles à tous (il n'existe pas de liste d'attente). Les membres de la communauté sont consultés régulièrement par l'intermédiaire d'enquêtes ou de réunions pour décider des types et des horaires des programmes et des activités. Le programme dispose d'une bibliothèque; une ambiance francophone se développe au moyen d'affiches et de messages muraux en français. Les enfants peuvent bénéficier d'une variété de jeux animés en français par les employés. Les programmes parascolaires comprennent un goûter et sont offerts du lundi au vendredi de $15 \mathrm{~h} 30$ à $18 \mathrm{~h} 00$. Les parents, les adolescents et adolescentes et les jeunes mères monoparentales peuvent par ailleurs profiter de programmes offerts régulièrement ou de façon ponctuelle.

Les enfants sont aussi exposés à d'autres éléments de la culture francophone, en assistant, notamment, à des pièces de théâtre, des spectacles ou des représentations d'artistes francophones. Les différentes fêtes francophones sont soulignées et parents et enfants y participent. Les programmes permettent aussi aux enfants de passer du temps avec des adultes dans un environnement sécuritaire.

La nature des activités offre donc aux enfants un climat contribuant à sauvegarder leur culture. Les jeunes découvrent qu'il 
"Les jeunes découvrent qu'il est possible de s'amuser dans leur langue maternelle...» est possible de s'amuser dans leur langue maternelle, comme le disent les employés du projet: «...les enfants doivent comprendre qu'ils peuvent étudier, s'amuser, interagir et aussi avoir du fun en français.»

Après la création de conditions favorisant pour les membres l'exposition aux autres cultures au moyen de réunions et d'ateliers, le problème de l'isolement culturel peut quand même survenir. La mise sur pied pendant l'été 1992 d'un programme hebdomadaire commun entre tous les enfants du projet n'atteint pas les objectifs de rapprochement visés. Les employés francophones constatent que, lors de ce programme, les enfants francophones s'expriment en anglais. Donc, sous la pression du groupe francophone, cette tentative est rapidement abandonnée, ce qui n'est pas sans soulever de nouvelles questions sur le rôle de la langue dans la culture. Les francophones font reconnaitre que, pour les enfants, l'usage de leur langue reste la première étape pour éviter l'assimilation.

C'est en septembre 1992 lors de l'atelier Naming the moment organisé par une agence locale et Partir d'un bon pas sur le thème de la participation communautaire, que la position francophone est clairement articulée. Pendant cet atelier, le groupe francophone affirme qu'il serait éventuellement intéressé à des programmes conjoints entre les cultures, mais qu'il n'est pas encore prêt à s'intégrer totalement aux autres groupes:

"On n'est pas encore prêt, nous ne voulons pas nous intégrer, l'intégration est un objectif à long terme. Nous ne fermons pas les portes, on est ouvert mais nos enfants ont besoin de plus long terme pour leur culture. La fierté d'être francophone n'est pas facile à développer chez nos enfants. Entre adultes on peut être ensemble, mais pour les enfants, pas maintenant.»

Cet atelier permet aussi de préciser certains objectifs particuliers du groupe pour restaurer la fierté francophone. Ces objectifs gravitent autour des points suivants: créer une place, reconnue comme Centre francophone, où les enfants peuvent apprécier leurs racines; encourager chez les enfants la fierté de leur 
Reflets

"...les francophones,

quant à eux, se définissent comme groupe séparé d'abord, l'intégration n'étant qu'un objectif à long terme.» culture francophone; développer et appuyer la créativité et le talent artistique des enfants; appuyer les artistes francophones; susciter une plus forte implication des parents et des personnes âgées; travailler à des projets communs par choix, pas de façon forcée.

Pendant cet atelier, les différences de vision entre les cultures démontrent que les anglophones sont prêts à travailler ensemble, tout comme les autochtones qui sont néanmoins seuls de temps en temps; les francophones, quant à eux, se définissent comme groupe séparé d'abord, l'intégration n'étant qu'un objectif à long terme.

Malgré la frustration engendrée chez les trois autres groupes culturels, cette mise au point permet de préciser que les intérêts ne sont pas divergents, mais que le processus necéssite patience et temps pour que chacun soit à l'aise chez soi avant de s'ouvrir aux autres.

En raison des limites financières, et du fait que les bailleurs de fonds du projet n'aient pas prévu une approche culturelle séparée, les francophones doivent se donner des moyens financiers pour atteindre certains de leurs objectifs. Le groupe francophone développe donc, à l'instar des autres groupes du projet, plusieurs activités de prélèvement de fonds pour financer certaines de ses activités. Ceci facilite une collaboration accrue entre le groupe francophone et les autres agences, organisations et institutions francophones de la ville.

La difficulté de favoriser un développement séparé des différents groupes culturels tout en assurant une cohésion et une vision communautaire est toujours présente au sein du projet, fait qu'exprime bien un des membres:

"Si nous commençons par isoler les cultures, et si nous attendons trop longtemps, nos différences vont s'accentuer avant que nous commencions à regarder dans la même direction.»

Aujourd'hui, la peur de l'isolement ou de la «ghettoïsation» des différentes cultures est en train de changer grâce à une plus grande collaboration entre les employés du projet. En effet, les rencontres et les échanges entre les employés des différents programmes sont 
"...malgré les différences culturelles, la communauté partage les mêmes rêves et ambitions quant à l'avenir de ses enfants.» de plus en plus fréquents, et visent à encourager le partage d'expériences et les échanges possibles entre programmes. Des échanges hebdomadaires permettent aussi à un ou une employé autochtone de faire des activités avec les enfants francophones. Et de façon régulière, un employé francophone assiste aux réunions des employés des autres centres et vice versa.

La vision communautaire se développe lentement, axée sur la constatation que malgré les différences culturelles, la communauté partage les mêmes rêves et ambitions quant à l'avenir de ses enfants. Le rêve de l'établissement de relations plus saines entre les parents et les enfants, le désir que le projet soit dirigé et contrôlé par la communauté et qu'il valorise et donne du pouvoir aux individus permettent un plus grand rapprochement entre les cultures (Naming the moment, 1992, p.24).

\section{Unenouvelle dimension dans la complexe diversité}

L'expérience de Partir d'un bon pas démontre que les francophones qui vivent comme minorité, malgré leur isolement, ont moins peur d'affirmer leur différence. Mais l'impact de l'appartenance familiale, du métier ou de la profession sur la réalité quotidienne des différentes communautés franco-ontariennes (Jaenen,1993), ainsi que l'appartenance communautaire et les mythes jouent un rôle de plus en plus important sur la cohésion ou la division de la communauté, comme le soutient un membre du projet:

"...C'est vrai en moyenne que des gens à faible re-
venu vivent dans cette communauté, mais il y en a
qui travaillent dans les mines et ont de bons salaires
mais sont vus comme des pauvres. Ce sont des gens
pauvres qui ont des difficultés à comprendre la réalité
de leur communauté qui véhiculent ces mythes. Il n'y
a pas que des pauvres au Moulin à Fleur, l'étiquette
vient de la division des francophones.»

Ayant seulement insisté dans ce texte sur le développement du caucus francophone, il faut savoir que tous les différents caucus du projet ont connu des développements différents malgré la vi- 
Reflets

"Le caucus

multiculturel... occupe

aujourd'hui un rôle

central dans la vie du

projet.» sion commune basée sur les objectifs du projet.Aujourd'hui, après trois ans d'existence, le projet a évolué vers une toute nouvelle structure qui maintient encore la dimension culturelle comme centre névralgique. Depuis l'hiver 1994, le caucus des agences qui a joué le rôle fondamental pendant la première année du projet a été complètement éliminé. Le caucus multiculturel qui pendant les deux premières années a eu de la difficulté à se constituer, occupe aujourd'hui un rôle central dans la vie du projet.

Depuis le mois d'avril 1994, le projet s'est doté d'une nouvelle structure qui a mené à son incorporation et à son indépendance visà-vis de son parrain administratif. Cette nouvelle structure est basée sur la réhabilitation des caucus, à l'exception de celui des agences, et la création d'un nouveau caucus pour les employés du projet. Plus d'une centaine de résidents et de sympathisants sont devenus membres du projet et ont élu un "Conseil» de 13 membres. Ce nouveau conseil élu remplace l'ancienne Association et joue le rôle d'un conseil d'administration (C.A.) pour le projet.

L'innovation vient d'abord du fait qu'en plus d'être regroupés au sein de caucus, les membres sélectionnent le caucus sur lequel ils veulent siéger (exception faite des employés qui sont obligés de siéger sur le caucus des employés). L'une des raisons pour cette nouvelle approche est qu'avec le développement du projet, le classement des membres dans une seule catégorie devenait de plus en plus difficile. Par exemple, un membre pouvait choisir d'être classé comme employé, ou simplement membre de la communauté francophone ou multiculturelle parce qu'il était originaire d'un pays francophone.

Les cinq caucus actuels (multiculturel, anglophone, autochtone, francophone et des travailleurs) élisent chacun deux membres pour siéger au C.A.; les trois autres membres sont élus par l'assemblée générale. Deux des trois membres ainsi élus proviennent de la communauté multiculturelle et le troisième est Autochtone. Ceci renforce la présence multiculturelle et crée une toute nouvelle dynamique au sein du projet. Les anglophones, le groupe qui faisait craindre l'assimilation et la perte de contrôle, sont maintenant devenus minoritaires, car seulement trois de leurs 
«...les différences

culturelles ont cessé

d'être une source de tension et de méfiance pour devenir une force de cette communauté.» représentants siègent au C.A. de 13 membres. En plus, étant donné que certains membres élus de la communauté multiculturelle sont francophones, les relations linguistiques et culturelles évoluent constamment.

La prééminence des anglophones est toujours évidente par le fait que les réunions du C.A. se déroulent encore en anglais. Cependant, lors des réunions, les participants sont de plus en plus ouverts aux autres cultures. Les cérémonies d'ouverture des deux dernières assemblées générales se sont faites selon les traditions autochtones, et le français ou l'anglais peut être utilisé pour communiquer. Avec ces nouveaux changements, on semble avoir assisté à un revirement de situation: les différences culturelles ont cessé d'être une source de tension et de méfiance pour devenir une force de cette communauté.

Dans cette nouvelle situation, la crainte des francophones visà-vis la domination anglophone est devenue moins importante, et une plus grande cohésion est en train de se développer au sein de ce groupe. Depuis quelques temps, on remarque d'ailleurs que les caucus ont des difficultés à fonctionner en dehors de la période des assemblées générales. Les résidents et résidentes rejoignent le projet de plus en plus comme membre de la communauté, et non forcément comme membre d'un groupe culturel. La vision commune comme membre d'une même communauté subissant l'oppression d'un système extérieur est en train de prendre le pas sur les difficultés d'intégration culturelle du début. Le désir de mettre l'accent sur la protection de la communauté fait dissiper les problèmes culturels même s'ils n'ont pas totalement disparu.

Après les trois premières années du projet, le caucus francophone a pu de son côté établir de solides programmes, donner une image francophone à son centre et établir des liens avec les organismes francophones de la région. Les francophones du projet sont donc aujourd'hui moins préoccupés par l'assimilation, et travaillent plus à la diversification de leurs activités qu'à la recherche de l'autonomie. Dans cette nouvelle structure, c'est le caucus multiculturel qui cherche à se protéger et à prendre sa place, ce qui situe le caucus francophone non plus comme un groupe minoritaire mais comme membre égal et puissant au sein du projet. 
Reflets

"...Il faut être fier de là où tu vis, il faut essayer de changer les choses, ce n'est pas en s'en allant qu'on va améliorer les choses.»
Les nouvelles tensions qui apparaissent sont maintenant liées à l'affectation des fonds entre les différents programmes. En effet, le développement du projet a entraîné une multiplication des programmes dans trois grands centres. Un troisième centre a été ouvert en octobre 1993 pour rejoindre d'autres membres de la communauté dont l'accès aux deux premiers centres était difficile en raison d'éloignement (les programmes dans ce nouveau centre sont en anglais). Cette situation rend la répartition des ressources disponibles très difficile et crée parfois de la compétition entre les programmes. Ceci est une cause de tension entre les groupes, sans toutefois être de nature strictement culturelle.

Dans une autre perspective, cette nouvelle dimension soulève au sein du groupe francophone la question de sa propre recomposition à cause du nouveau visage de la francophonie ontarienne. Le statut des autres minorités francophones reste très aléatoire dans la mesure où elles rencontrent parfois des problèmes d'intégration dans le groupe multiculturel. L'obstacle linguistique se dresse comme une forte barrière, en particulier dû au fait que certains de leurs membres sont souvent unilingues francophones.

Au sein du groupe francophone lui-même, des difficultés peuvent aussi surgir à long terme. Certains francophones du site pensent qu'il y a parfois une attitude de fuite vers l'extérieur chez les membres de la communauté qui considèrent le Moulin à Fleur comme un taudis, un «trou». Ils estiment qu'il y a un travail à faire pour changer les attitudes négatives de certains membres de la communauté ainsi que celles de certains organismes francophones dont la collaboration reste plutôt minime. Comme le dit un membre: "...Il faut être fier de là où tu vis, il faut essayer de changer les choses, ce n'est pas en s'en allant qu'on va améliorer les choses.»

L'attitude du groupe francophone face aux organismes institutionnels pose le problème de la solidarité des organisations francophones. La bonne majorité des organismes, même s'ils ne peuvent qu'appuyer le développement du projet, ne s'impliquent pas ou y contribuent très peu. L'attitude de classe est parfois mentionnée comme une des raisons, comme le dit si bien un membre du 
projet: «il y a une bourgeoisie, une élite francophone qui refuse de s'impliquer, l'éducation est la cause de la division de classe».

Ces situations posent donc de nouveaux problèmes auxquels la communauté francophone doit faire face. Cependant, le ton est donné pour une approche dans laquelle les différentes communautés culturelles du site commencent à se rapprocher pour relever certains défis communs, c'est-à-dire la pauvreté, les mythes, le statut de classe et l'attitude négative de certaines institutions.

L'une des manifestations les plus importantes du renforcement de cette nouvelle solidarité a été de voir, pendant l'hiver et le printemps 1994, les différents membres du projet travailler ensemble sans aucune barrière culturelle, pour lutter contre le welfare snitch line (dénonciation des fraudes contre l'assistance sociale). Tous les membres du projet considéraient cette politique comme un élément de destruction et de désorganisation de leur communauté, car elle créait des soupçons entre les résidents et résidentes.

Dans cette phase donc, la notion de minorité est en train de se transformer pour céder la place à une nouvelle solidarité, et celle-là est basée moins sur la culture que sur l'appartenance sociale.

\section{En guise de conclusion}

L'expérience de la communauté francophone du projet Partir d'un bon pas de Sudbury est intéressante à plus d'un titre. On a pu constater qu'un groupe s'étant constitué sur la base d'une minorité défensive se retrouve, après trois ans d'évolution, dans une structure au sein de laquelle il joue un rôle prépondérant. Il est vrai que dans ce texte l'accent a été mis sur le groupe francophone, mais la dynamique du projet a permis une interaction culturelle dans laquelle les quatre groupes culturels représentant la communauté du Moulin à Fleur/Donovan ont développé des stratégies leur ayant permis d'atteindre des objectifs spécifiques et communs. 
Reflets

À Sudbury, vit une communauté franco-ontarienne de souche sudburoise, mais leur réalité a beaucoup changé ces dernières années avec la montée du conformisme, face à la culture dominante, et à cause de l'arrivée et de l'installation dans ce quartier d'une communauté multiculturelle qui s'assimile plus facilement à la culture dominante. Il a donc fallu à la communauté francophone une structure lui permettant d'affirmer sa différence afin de rompre le cycle de l'assimilation et de protéger, voire assurer, le développement culturel des prochaines générations.

Plusieurs facteurs inhérents à la structure et au mode de fonctionnement du projet ont contribué à l'atteinte des objectifs de développement culturel et communautaire de ce projet. D'abord la création des caucus sur une base culturelle a permis d'assurer une certaine autonomie des différents groupes et a contribué à l'affirmation de leurs particularités. Grâce à ce facteur, les différentes communautés culturelles ont pu s'organiser de l'intérieur avant de s'ouvrir aux autres. Ceci rejoint l'idée de Roger Bernard (1986) lorsqu'il écrit que:

"Les institutions ethniques doivent encadrer de nombreuses pratiques sociales, favoriser les échanges à l'intérieur du groupe et ainsi participer à la construction de la communauté franco-ontarienne et au développement d'un champ culturel d'une génération à l'autre."

La capacité de choisir son propre caucus a permis, dans l'étape la plus récente du projet, de redéfinir la réalité culturelle dans la communauté, favorisant ainsi une plus grande ouverture. La catégorisation des individus semble de moins en moins répondre à l'approche interculturelle du projet. Le nouveau visage de la francophonie ontarienne avec les immigrants francophones commence à avoir un impact sur le projet. Après une période pendant laquelle les membres avaient besoin d'une identification culturelle pour marquer leur différence, le projet est maintenant dans une phase d'ouverture à l'intégration sans assimilation. Il faudrait cependant noter que le groupe francophone a pu imposer un rythme qui lui a permis de se préparer et de ne pas avoir à se 
soumettre à l'intégration. Le temps, ainsi que la double structure caucus/Association, ont donc permis au groupe francophone de travailler de l'intérieur et de définir un terrain propre tout en participant aux objectifs collectifs.

Parmi les éléments qui ont facilité l'atteinte de ces objectifs, il faut aussi considérer le processus de prise de décision. Le choix du consensus comme moyen décisionnel a favorisé et a donné le temps aux différents groupes culturels de se connaître, de se concerter et de s'engager dans des décisions après de longues discussions. Ceci a encouragé la participation de tous les groupes culturels dans des décisions d'importance. Ces temps de réflexions et de discussions ont été favorables à une meilleure compréhension entre les différents acteurs. À cela vient s'ajouter l'éducation multiculturelle constante encouragée par l'entremise d'ateliers de formation. Ceci nous amène à nous demander si on aurait pu atteindre les mêmes résultats au moyen d'une autre forme de prise de décision.

On peut aussi mentionner les procédures d'embauche qui ont permis aux différentes communautés de participer au choix de leurs travailleurs et travailleuses, et d'avoir leur mot à dire sur la sélection des autres employés du projet. Dans de telles situations, la minorité possède un grand avantage de négociation, puisque le groupe hésite généralement à déclencher un véritable processus de séparation ou de rejet (Moscovici, 1979). Dans plusieurs cas, donc, les francophones ont pu se faire entendre et on a pu atteindre un consensus.

En embauchant des membres de la communauté pour plus de $90 \%$ des postes, le projet a favorisé à long terme le développement d'une conscience communautaire au-delà de l'appartenance culturelle. De plus, les rencontres régulières entre les employés ont entraîné des échanges de méthodes de travail et ont favorisé une meilleure collaboration et compréhension interculturelles.

Le mode de gestion choisi par le projet a donc favorisé l'autogestion des différents programmes et ceci a permis un réel transfert de certains pouvoirs décisionnels au niveau des employés et 
Reflets

"Le modèle de Sudbury a permis aux membres de communautés défavorisées de travailler ensemble à la restauration de leur fierté culturelle.» des centres. Chaque caucus a donc pu décider des activités susceptibles de les aider à promouvoir leur culture et à protéger leurs enfants contre l'assimilation, par la socialisation qui permet de partager une culture, une identité et une histoire (Bernard, 1986) en dehors de l'école.

Le modèle de Sudbury a permis aux membres de communautés défavorisées de travailler ensemble à la restauration de leur fierté culturelle. La culture a été utilisée dans cette dynamique comme une force et un moyen pour le groupe francophone de se constituer et de s'affirmer. Malgré la pauvreté dans leur quartier, l'affirmation de l'identité culturelle des membres de la communauté nous montre que l'approche choisie a permis de reconstituer la motivation et l'énergie collective et d'aider à mieux asseoir les identités et les différences.

En choisissant l'approche interculturelle, le projet est parvenu à dissiper la méfiance quant à la domination et à l'assimilation; ceci a permis aussi aux membres de la communauté de travailler ensemble à des objectifs spécifiques et communs. Ceci a aussi contribué à donner aux communautés impliquées la chance d'apprendre au sujet des autres cultures, de développer de nouvelles habiletés et de construire une collectivité forte et fière. Cette approche a permis une concertation qui a fortifié les différentes cultures et leur a permis d'être plus en mesure, de façon séparée ou collective, de faire face aux forces oppressives. En même temps, elle a permis de donner des sources quotidiennes de joie, d'affirmation, de célébration et de valorisation des rituels aux parents et aux enfants.

Avec l'éclatement politique du Canada français, les FrancoOntariennes et Franco-Ontariens vivant dans des milieux défavorisés sont-ils en train de prendre meilleure conscience de leur identité? Étant donné qu'une forte proportion de FrancoOntariennes et Franco-Ontariens vivent au-dessous du seuil de pauvreté (Savard, Pierre, 1990), quel est l'incidence de ce facteur sur la lutte pour le maintien de l'identité? On peut déjà dire que l'expérience du projet Partir d'un bon pas démontre que la volonté 
"...le groupe doit se valoriser de l'intérieur à partir d'éléments qui débordent le simple fait de l'apprentissage d'une langue.» et le pouvoir sont encore très forts même dans les communautés dites défavorisées. Au dire d'un membre,

"Mon rêve est que le projet réussisse à faire en sorte que les jeunes voient l'importance de garder la langue, de découvrir la culture, de s'affirmer positivement, d'avoir la motivation pour prouver qu'ils peuvent réussir même s'ils viennent d'un milieu moins favorisé."

Tout ceci nous pousse aussi à nous questionner sur la théorie selon laquelle la conscience d'appartenance culturelle chez les francophones de l'Ontario est un phénomène qui se développe par le haut, c'est à dire à partir des intellectuels (Laflamme, 1990). À travers ce projet, on voit se développer une forte conscience d'appartenance culturelle dans des populations défavorisées.

L'expérience de Sudbury démontre que la situation de minorité ne fait pas forcément d'une communauté culturelle un groupe soumis. L'affirmation de sa différence et de sa fierté n'est cependant possible que lorsqu'il existe des zones de collaboration, de complémentarité afin de réduire les tensions liées à l'incompréhension. L'affirmation de l'identité culturelle des FrancoOntariens à travers le projet a montré que le groupe doit se valoriser de l'intérieur à partir d'éléments qui débordent le simple fait de l'apprentissage d'une langue (Laflamme, 1990). La dynamique au sein du projet a permis à chaque groupe culturel de manifester sa différence au niveau des besoins, des priorités et même des méthodes de travail. Cependant, c'est à travers une ouverture aux autres cultures qu'une certaine cohésion et cohabitation entre cultures ont été atteintes.

Dans ce contexte, le rôle des institutions et des projets communautaires devrait être de faciliter et de créer des voies permettant d'intégrer les différences dans une vision commune, et d'éviter de forcer une intégration culturelle qui conduit souvent à l'assimilation des minorités. «Partir d'un bon pas» de Sudbury essaie de trouver des solutions à l'intégration culturelle, mais plusieurs questions trouveront des réponses à long terme puisque le projet existe seulement depuis trois ans et que, d'ici 1997, la situation aura bien le temps d'évoluer et de changer. Dossier à suivre... 


\section{Bibliographie}

ALARY, Jacques (1990). Community Care and Participatory Research, traduit par Susan Usher, Montréal, $\mathrm{Nu}-$ Age Éditions.

ANGERS, Pierre (1960). Problèmes de culture au Canada français, Montréal, Éditions Beauchemin.

ARSENAULT, Robert (1993). «Alphabétiser la jeunesse franco-ontarienne: Quels défis à l'horizon pour l'AOPF?», Revue du Nouvel-Ontario, no 13-14, pp. 89-106.

BERNARD, Roger (1986). «Le rôle social des institutions ethniques», Revue du Nouvel-Ontario, no 8, pp. 41-48.

BERNARD, Roger (1994). «Du social à l'individuel: naissance d'une identité bilingue», dans Jocelyn Létourneau, dir. La question identitaire au Canada francophone, Québec, Les Presses de l'Université Laval, pp. 155-162.

BRAËN, André (1986). «Langue, droit et institutions autonomes» - Les mots et les choses, Revue du Nouvel-Ontario, no 8, pp. 29-39.

CARRIÈRE, Richard, C. HEIN, M. REITSMA-STREET et A.VAN DE SANDE (1992). La pauvreté chez les enfants et chez les jeunes dans la région de Sudbury, Sudbury, École de Service Social, Université Laurentienne.

COMER, James P. (1980). School Power-Implication of an Intervention Project, New York, The Free Press.

CORNELIUS, J. Jaenen (1993). Les Franco-Ontariens, Ottawa, Les Presses de l'Université d'Ottawa, pp. 3-6

DESLAURIERS, Omer (1989). «La situation de la vie franco-ontarienne», Revue du Nouvel-Ontario, no 1 , pp. 23-32.

DENNIE, Donald, (1978). «De la difficulté d'être idéologue francoontarien», Revue du NouvelOntario, no 1, pp. 69-90.

GUINDON, René (1984). «Remarques sur la communauté franco-ontarienne comme entité politique", Revue du Nouvel-Ontario, no. 6, 49-64.

HEIN, Carol (1994). "Poverty Rates for Children, Youth and Families in Sudbury region», dans Marge Reitsma-Street, ed. Child and Young Poverty in Sudbury: A Follow Up Report, Sudbury, School of Social Work, Laurentian University, pp. 8-26.

JOHNSTON, Patrick (1983). Native Children and the Child Welfare System, Toronto, The Canadian Council on Social Development in association with James Lorimer and Company, Publishers.

KITCHEN, Brigitte, A. MITCHELL, P. CLUTTERBUCK et M. NOVIC (1991). Unequal Futures: The Legacies of Child Poverty in Canada, Toronto, The Child Poverty Action Group \& The Social Planning Council of Metropolitan Toronto.

LAFLAMME, Simon, D. DENNIE et Y. GAUTHIER (1990). L'ambition démesurée: enquête sur les étudiants et étudiantes francophones du Nord-est de l'Ontario, Sudbury, Éditions Prise de parole.

LIPMAN E.L., D.R. OFFORD et M.H. BOYLE (1994). «Relation Between Economic Disavantage and Psychosocial Morbidity in Children", Canadian Medical Association Journal, vol. 151, no 4, pp. 431-437.

MCFATE, Katherine (1991). Poverty, Inequality and the Crisis of Social Policy, Washington, D.C., Joint Centre for Political and Economic Justice. 
MINISTÈRE DES SERVICES SOCIAUX ET COMMUNAUTAIRES (1989). Partir d'un bon pas pour un avenir meilleur: un modèle intégré de prévention primaire des difficultés affectives et comportementales, Toronto, Imprimeur de la Reine pour l'Ontario.

MOSCOVICI, Serge (1979). Psychologie des minorités actives, Paris, Presses Universitaires de France.

OFFORD, David R., Micheal H. BOYLE et B.R. JONES (1987). «Psychiatric Disorder and Poor School Performance Among Welfare Children in Ontario", Canadian Journal of Psychiatry, vol. 32, pp. 518-525.

PILON, Claire (1983). Le Moulin à Fleur, Sudbury, Les Presses ACME Ltée.

PETERS, Ray Dev et Crill Carol RUSSEL (1994). Le projet Partir d'un bon pas pour un avenir meilleur: vue d'ensemble du modèle, des programmes et de la recherche, Toronto, Imprimeur de la Reine pour l'Ontario.

REITSMA-STREET Marge, M. CARRIÈRE, R. HEIN et C.VAN DE SANDE (1993). «Three Perspectives on Child Poverty in Canada», The Social Worker, vol. 61, no 1, pp. 6-12.

RUTTER, Michael et MADGE, Nicola (1976). Cycles of Disadvantage: Studies in Deprivation and Disadvantage, London, Heinemann.

RUTTER, Micheal (1983). «School Effects On Pupil Progress», Child Development, vol. 54, pp. 129.

SAVARD, Pierre (1990). «De la difficulté d'être Franco-Ontarien», Revue du Nouvel-Ontario, no 1, pp. 11-22.

SERVICE DE SANTÉ MENTALE POUR ENFANTS (1992). Naming the Moment Together: Notes in Progress, Sudbury, Le Service de Santé Mentale pour enfants à Sudbury.

SMEEDING, T. (1988/89) «Poverty, Affluence and Income Costs of Children: Cross-National Evidence from the Luxembourg Income Study", Journal of Post-Keynesian Economics, Winter, pp. 222-239.

STATISTIQUE CANADA (1987). Statistique Canada: Secteurs de recensement - Sudbury - Partie 2, Ottawa, ministère des Approvisionnements et Services Canada.

VOLPÉ, Richard (1989). La pauvreté et l'enfance maltraitée: Une analyse de documents choisis, Toronto, University of Toronto, Institute for Child Studies. 